Western University

Scholarship@Western

$3-7-2009$

\title{
Analysis of carotid lumen surface morphology using three- dimensional ultrasound imaging
}

\author{
Bernard Chiu \\ Vadim Beletsky \\ J David Spence \\ Western University, dspence@robarts.ca \\ Grace Parraga \\ Aaron Fenster
}

Follow this and additional works at: https://ir.lib.uwo.ca/biophysicspub

Part of the Medical Biophysics Commons

\section{Citation of this paper:}

Chiu, Bernard; Beletsky, Vadim; Spence, J David; Parraga, Grace; and Fenster, Aaron, "Analysis of carotid lumen surface morphology using three-dimensional ultrasound imaging" (2009). Medical Biophysics Publications. 130.

https://ir.lib.uwo.ca/biophysicspub/130 


\section{Analysis of carotid lumen surface morphology using three-dimensional ultrasound imaging}

To cite this article: Bernard Chiu et al 2009 Phys. Med. Biol. 541149

View the article online for updates and enhancements.

\section{Related content}
Novel 3D ultrasound image-based biomarkers based on a feature selection from a 2D standardized vessel wall thickness map: a tool for sensitive assessment of therapies for carotid atherosclerosis
Bernard Chiu, Bing Li and Tommy W S Chow
- Quantification and visualization of carotid segmentation accuracy and precision using a 2D standardized carotid map Bernard Chiu, Eranga Ukwatta, Shadi Shavakh et al.
3D ultrasound for lung tumours V D M Hornblower, E Yu, A Fenster et al.

\section{Recent citations}

- Fractal dimension based carotid plaque
$\frac{\text { characterization from three-dimensional }}{\text { ultrasound images }}$
Ran Zhou et al
- Fractal analysis of plaque border, a novel
$\frac{\text { method for the quantification of }}{\text { atherosclerotic plaque contour irreqularity }}$
$\frac{\text { is associated with pro-atherogenic plasma }}{\text { lipid profile in subjects with non-obstructive }}$
$\frac{\text { carotid stenoses }}{\text { Francesco Moroni et al }}$
- Correspondence optimization in 2D
$\frac{\text { standardized carotid wall thickness map by }}{\text { description length minimization: A tool for }}$
$\frac{\text { increasing reproducibility of 3D ultrasound- }}{\text { based measurements }}$
Yimin Chen and Bernard Chiu




\title{
Analysis of carotid lumen surface morphology using three-dimensional ultrasound imaging
}

\author{
Bernard Chiu $^{1,2}$, Vadim Beletsky ${ }^{1,3}$, J David Spence ${ }^{1,3}$, Grace Parraga ${ }^{1,2}$ \\ and Aaron Fenster ${ }^{1,2}$ \\ ${ }^{1}$ Imaging Research Laboratories, Robarts Research Institute, London, Ontario, Canada \\ 2 Graduate Program in Biomedical Engineering, University of Western Ontario, London, \\ Ontario, Canada \\ ${ }^{3}$ Stroke Prevention and Atherosclerosis Research Centre, Robarts Research Institute, London, \\ Ontario, Canada \\ E-mail: bcychiu@alumni.uwo.ca,vados@imaging.robarts.ca,dspence@robarts.ca, \\ gep@imaging.robarts.ca and afenster@imaging.robarts.ca
}

Received 10 October 2008, in final form 8 December 2008

Published 27 January 2009

Online at stacks.iop.org/PMB/54/1149

\begin{abstract}
Carotid plaque surface irregularity and ulcerations play an important role in the risk of ischemic stroke. Ulcerated or fissured plaque, characterized by irregular surface morphology, exposes thrombogenic materials to the bloodstream, possibly leading to life- or brain-threatening thrombosis and embolization. Therefore, the quantification of plaque surface irregularity is important to identify high-risk plaques that would likely lead to vascular events. Although a number of studies have characterized plaque surface irregularity using subjective classification schemes with two or more categories, only a few have quantified surface irregularity using an objective and continuous quantity, such as Gaussian or mean curvature. In this work, our goal was to use both Gaussian and mean curvatures for identifying ulcers from 3D carotid ultrasound (US) images of human subjects. Before performing experiments using patient data, we verified the numerical accuracy of the surface curvature computation method using discrete spheres and tori with different sampling intervals. We also showed that three ulcers of the vascular phantom with $2 \mathrm{~mm}, 3 \mathrm{~mm}$ and $4 \mathrm{~mm}$ diameters were associated with high Gaussian and mean curvatures, and thus, were easily detected. Finally, we demonstrated the application of the proposed method for detecting ulcers on luminal surfaces, which were segmented from the 3D US images acquired for two human subjects.
\end{abstract}

(Some figures in this article are in colour only in the electronic version) 


\section{Introduction}

Stroke is the most common serious neurological problem globally and the third leading cause of death among North American adults (Thom et al 2006, The Heart and Stroke Foundation of Canada 2003). The direct and indirect costs of stroke alone are estimated to be $\$ 2.8$ billon year $^{-1}$ in Canada and $\$ 51$ billon year $^{-1}$ in the USA (Thom et al 2006, The Heart and Stroke Foundation of Canada 2000, The Heart and Stroke Foundation of Canada 2003). Clearly, stroke represents a staggering mortality, morbidity and economic cost. Improved methods for identifying patients at increased risk of stroke and better techniques for treating and monitoring them will have an enormous impact.

About $85 \%$ of strokes are ischemic, mostly caused by the blockage of a cerebral artery by a thrombotic embolus. Atherosclerosis at the carotid bifurcation is a major cause for the generation of thrombosis and subsequent cerebral emboli (Golledge et al 2000). Improved identification of patients who are at risk of stroke, new strategies for treating atherosclerosis and sensitive techniques for monitoring carotid plaque in response to therapy will have a great impact on the management of these patients, and decrease the risk of stroke.

Degree of stenosis has been used as the conventional clinical measure of severity of atherosclerosis in the carotid arteries. However, the degree of stenosis alone is not a sufficiently sensitive and specific marker of the risk of stroke. The NASCET results showed that $74 \%$ of the patients with severe stenosis $(\geqslant 70 \%)$ do not suffer stroke within two years (Barnett 1992), and ECST results showed that $10 \%$ of symptomatic patients with low-grade stenosis (17-50\% by NASCET) suffer ipsilateral stroke within four years (Warlow 1991). Although the risk of stroke increases with the severity of carotid stenosis (NASCET Collaborators 1991, Warlow 1991), it is generally accepted that the risk of major vascular events is more related to the stability of plaque than to carotid stenosis (Eliasziw et al 1994, Streifler et al 1994, Spence et al 2005, Fisher et al 2005). Ulcerated or fissured plaque, characterized by irregular surface morphology, exposes thrombogenic materials to the bloodstream, possibly leading to life- or brain-threatening thrombosis and embolization (Sztajzel 2005, Fisher et al 2005, Rothwell et al 2000, Sitzer et al 1995). Therefore, the ability to characterize and quantify plaque surface irregularity may improve the efficacy of stroke prevention by carotid endarterectomy (Fisher et al 2005).

Several investigations using $\mathrm{x}$-ray angiography analysed plaque ulcerations by classifying them into two or three categories qualitatively and subjectively (e.g., ulcerated/irregular, no ulceration/smooth and uncertain), and then correlated these classifications with occurrence of vascular events (Eliasziw et al 1994, Streifler et al 1994, Rothwell et al 2000). Other investigations have used ultrasound imaging for identifying plaque ulcerations (Steinke et al 1992, Sitzer et al 1996, Schminke et al 2000, Sztajzel 2005). In these studies, plaque ulcerations were defined as recesses with a certain depth (e.g., $2 \mathrm{~mm}$ in Schminke et al (2000), Sztajzel (2005)), and with a well-defined back wall at the base exhibiting an area of reverse flow as shown in power Doppler imaging. Miskolczi et al (1996) gave a more specific definition of plaque ulceration in their study using intravascular ultrasound. They also set the lower limit of the orifice diameter and depth of an ulceration to $0.5 \mathrm{~mm}$, which they measured for each suspected ulceration from the intravascular ultrasound image. However, the above studies did not further quantify the degree of surface irregularity of the detected ulcerations.

Mean and Gaussian curvatures are two local geometric descriptors of surface 'roughness' in classical differential geometry. Differential geometric theories for continuous surfaces are well established (do Carmo 1976). However, there is no consensus on the best method for estimating surface curvatures on discrete surfaces, although the triangular mesh representation of surfaces is extensively used in the computer graphics community (Meyer et al 2003). 
Previous investigations using 3D ultrasound (US) and magnetic resonance (MR) imaging techniques have reported quantification techniques for plaque surface morphology. Fenster et al (2004) reconstructed the segmented contours of plaque into a 3D surface mesh. They then calculated the local Gaussian curvature at each vertex and averaged the curvature values within a $1 \mathrm{~mm}$ radius of each vertex on the mesh. The results presented in Fenster et al (2004) are, however, preliminary and have not been validated. Han et al (2001) have computed the Gaussian curvature of the lumen surface that they reconstructed from contiguous contours segmented from MR images. The Gaussian curvature of each vertex on the mesh was computed using the method introduced in Besl and Jain (1986). However, the Gaussian curvature computation method by Besl and Jain (1986) ignored the surface partitioning issue, which is essential to establish a tight error bound for curvature estimation. In addition, a partitioning scheme without region overlapping is essential for the Gauss-Bonnet theorem, an important geometrical property, to be satisfied (Meyer et al 2003).

The goal of this paper is to identify ulcers from in vivo 3D US images based on the mean and Gaussian curvatures computed using the method proposed by Meyer et al (2003). Before achieving this goal, we demonstrate the accuracy of the surface curvature computation algorithm using synthetic surfaces and the practicality of the algorithm in detecting ulcers using a vascular phantom.

\section{Methods}

In this section, we describe the surface curvature computation algorithm, introduce three types of surface data that were used to validate the algorithm and describe how these data were obtained. The three types of surfaces are: (1) synthetic spherical and toridal surfaces, (2) surfaces constructed by segmenting a 3D US image of a vascular phantom with ulcermimicking hemispheres and (3) surfaces constructed from 3D US images of human subjects. The Gaussian and mean curvatures of the synthetic surfaces are known. The purpose of computing the curvatures of these surfaces was to verify the numerical accuracy of the algorithm. The purpose of computing the curvatures of the vascular phantom was to show that ulcers have higher mean and Gaussian curvatures, and thus, curvatures can be used as a feature to assist the detection of ulcers. Finally, we compute the surface curvatures for carotid lumen of human subjects to demonstrate that the purposed algorithm can be used to detect ulcers from $3 \mathrm{D}$ carotid ultrasound images.

\subsection{Vascular phantom with ulcerations}

To simulate the carotid vessel, a vascular phantom was constructed and filled with a heated $85^{\circ} \mathrm{C}$ agar mixture consisting of the following (by \% mass): $3 \%$ agar (A-7002, Sigma-Aldrich, Oakville, ON, Canada), $8 \%$ glycerol, $86 \%$ distilled water, 3\% Sigmacell ${ }^{\circledR}$ (S-5504, SigmaAldrich). Sigmacell ${ }^{\circledR}$ is a product consisting of $50 \mu \mathrm{m}$ cellulose particles added as scatterers (Rickey et al 1995). Hemispheres made of acetal with diameters of $2 \mathrm{~mm}, 3 \mathrm{~mm}$ and $4 \mathrm{~mm}$ (McMaster-Carr, Aurora, $\mathrm{OH}$ ) were glued on a brass cylinder with $10 \mathrm{~mm}$ diameter. The distance between the centres of the $2 \mathrm{~mm}$ and $3 \mathrm{~mm}$ hemispheres was $11 \mathrm{~mm}$, and the distance between the centres of the $3 \mathrm{~mm}$ and $4 \mathrm{~mm}$ hemispheres was $14 \mathrm{~mm}$ (figure 1(a)). The brass cylinder was composed of two longitudinal halves, which were inserted into a box to create a vascular channel that was $15 \mathrm{~mm}$ below the surface of the phantom. The agar mixture was then poured into the vascular phantom box and allowed to cool for 30-40 min until the agar mixture solidified. Then, the brass cylinder was removed. To avoid damaging the phantom, 


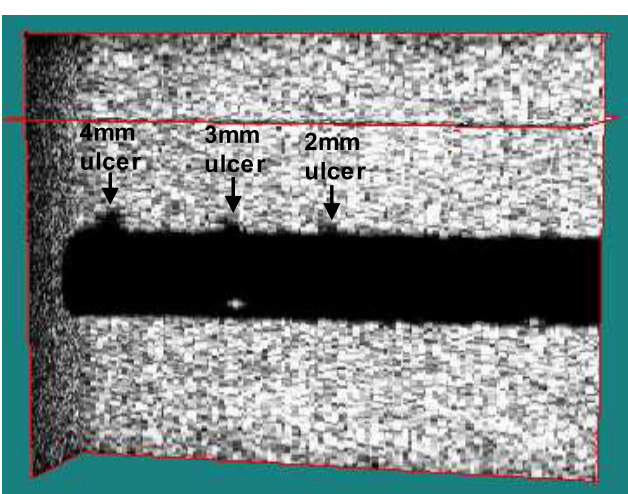

(a)

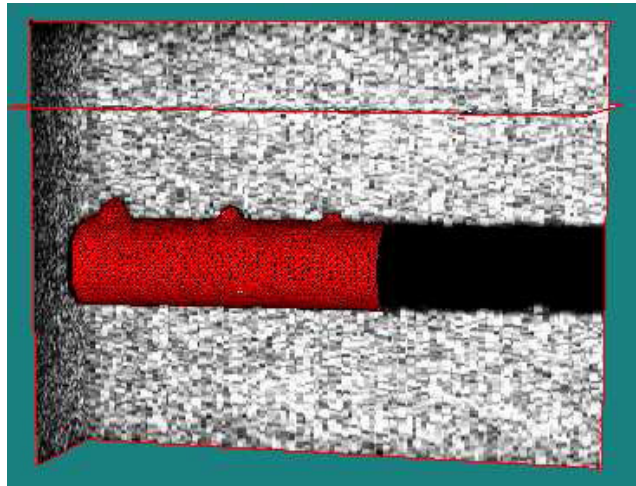

(b)

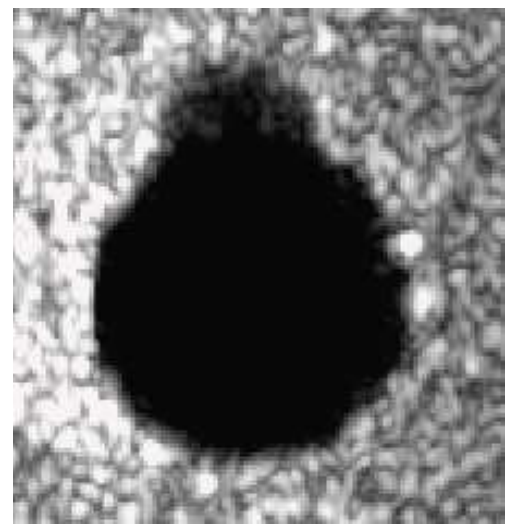

(c)

Figure 1. 3D ultrasound image of the phantom. (a) The longitudinal view of the phantom with the $2 \mathrm{~mm}, 3 \mathrm{~mm}$ and $4 \mathrm{~mm}$ ulcers labelled. (b) The same longitudinal view as shown in (a), but with the segmented luminal surface superimposed on the image. (c) The transverse view of the 4 mm ulcer.

the bottom half of the cylinder with no sphere was removed first. Then, the top half was detached from the phantom, before it was slid out.

\subsection{Image acquisition for the vascular phantom}

The 3D carotid US imaging system was described elsewhere (Fenster et al 2001) and is briefly summarized here. The images of the phantom were acquired by translating an ultrasound transducer (L7-4, ATL-Philips) over the surface of the phantom along the longitudinal axis of the vessel, with the three synthetic ulcers facing the transducer. The US probe was held by a mechanical assembly, and the transducer angle was fixed to be perpendicular to the phantom surface and the longitudinal axis of the vessel. The video frames from the US machine (HDI5000, ATL-Philips) were digitized, saved to a computer workstation and reconstructed into a 3D image (Fenster et al 2001, Fenster et al 2004, Landry and Fenster 2002, Landry et al 2005). The acquired $2 \mathrm{D}$ images were parallel to each other with a pixel size of $0.13 \mathrm{~mm} \times$ $0.13 \mathrm{~mm}$. The mean spatial interval between adjacent $2 \mathrm{D}$ images was of $0.3 \mathrm{~mm}$. 


\subsection{Study subjects and image acquisition}

We used the 3D US images acquired for two subjects to demonstrate the application of our algorithm. These subjects, who participated in a clinical study focusing on the effect of atorvastatin, were asymptomatic with carotid stenosis $>60 \%$ according to carotid Doppler flow velocities (Ainsworth et al 2005). Before conducting our study, we obtained consent from each of the subjects regarding the study's protocol, which was approved by the University of Western Ontario's standing board of human research ethics.

The 3D US images were acquired by translating an ultrasound transducer (L12-5, ATLPhilips) along the neck of the subjects for approximately $4.0 \mathrm{~cm}$, which takes approximately $8 \mathrm{~s}$. The transducer angle was fixed to be perpendicular to the skin and the direction of the scan for all patient scans. The US machine and the image pixel sizes were the same as those used for the phantom image acquisition.

\subsection{Carotid segmentation and surface reconstruction}

The vascular phantom was segmented using the semi-automatic segmentation algorithm developed by Ladak et al (2000) based on the discrete dynamic contour (DDC) model proposed by Lobregt and Viergever (1995). A transverse 2D image at approximately $8 \mathrm{~mm}$ away from the centre of the $2 \mathrm{~mm}$ hemisphere (i.e., $19 \mathrm{~mm}$ and $33 \mathrm{~mm}$ from the centres of $3 \mathrm{~mm}$ and $4 \mathrm{~mm}$ hemispheres, respectively) was chosen as the initial 2D image, and a single observer initialized four points, from which the boundary of the phantom was determined using a cubic-spline interpolation technique (Ladak et al 2000). The estimated boundary on the initial $2 \mathrm{D}$ image was then automatically refined using the DDC model. The deformed contour was then propagated to the adjacent $2 \mathrm{D}$ transverse image, which was $1 \mathrm{~mm}$ away from the initial $2 \mathrm{D}$ image, and was used as the initial contour before refining. This process was repeated until the complete vascular phantom was segmented.

From the 3D US images acquired for two subjects, ulcers on the CCA lumen were identified by a physician who is experienced in analysing carotid US images. A separate observer, knowing the location of the ulcers as identified by the physician, segmented the CCA lumen using the method described in the previous paragraph, for $10 \mathrm{~mm}$ with a $1 \mathrm{~mm}$ inter-slice distance.

Although it was reported that ulcers larger than $1 \mathrm{~mm}$ are clinically significant (Sitzer et al 1995) and that ulcers with orifice diameter and depth larger than $0.5 \mathrm{~mm}$ could be detected using intravascular US (Miskolczi et al 1996), non-invasive US imaging has a coarser resolution and the consensus is to define ulcerations as craters with a width and depth of $2 \mathrm{~mm}$ (de Bray et al 1997, Schminke et al 2000, Meairs and Hennerici 1999, Sztajzel 2005). By choosing $1 \mathrm{~mm}$ as the inter-slice distance, ulcerations with a width and depth of $2 \mathrm{~mm}$ will not be missed as the sampling interval is exactly half of the size of the ulcer. Inter-slice distance greater than $1 \mathrm{~mm}$ may lead to distortion of the shape of the ulcer.

Each set of 2D contours was then reconstructed into a triangulated surface mesh (Chiu et al 2006, 2008b). The reconstructed surface of the phantom is represented as a red surface superimposed on the 3D US image of the phantom in figure 1(b), and the luminal surfaces for the two subjects are shown in figures 6(b) and 7(b).

\subsection{Computation of surface curvatures}

Surface curvature computation methods can be classified into two major types. The first type is based on local surface fitting and the second type estimates the curvature directly from the triangular mesh (Besl and Jain 1986, Han et al 2001). Local surface fitting can be achieved by 


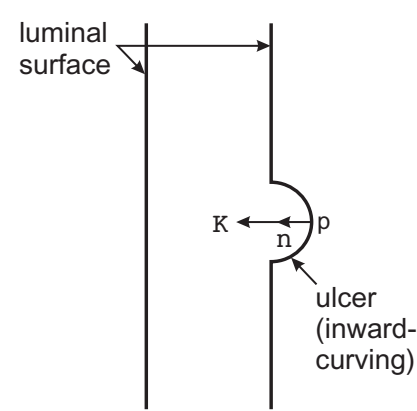

(a)

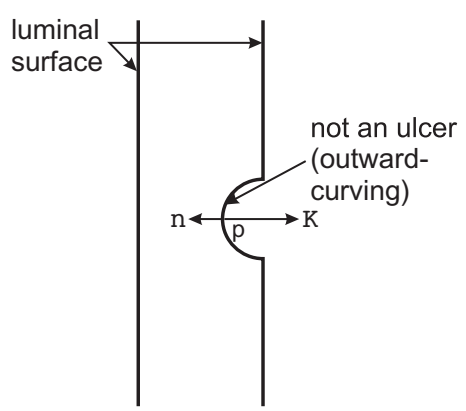

(b)

Figure 2. Two shapes that have the same Gaussian curvatures, but different mean curvatures. This figure shows that if high Gaussian curvature was used as the only criterion to identify ulcers, both the inward- and outward-curving regions, shown in (a) and (b) respectively, would be identified as ulcers. However, the outward-curving region shown in (b) is not an ulcer while (a) shows an ulcer. The mean curvature normal vector $\mathbf{K}(p)$ points inwards from the surface. Thus $\kappa_{H}(p)$ is positive according to equation (5). (b) shows a region that has the same Gaussian curvature as the ulcer shown in (a). Since it curves outwards, it is not an ulcer. $\mathbf{K}(p)$ points outwards from the surface, and $\kappa_{H}(p)$ is negative according to equation (5). Ulcers are associated with high Gaussian and mean curvatures.

polynomial reconstruction and analytical evaluation. However, overshooting and unexpected surface behaviour between sample points often occur with this method (Meyer et al 2003). With this in mind and that we have established a method for constructing a triangular mesh for a stack of segmented lumen contours in our previous work (Chiu et al 2006, 2008b), we used the algorithm proposed by Meyer et al (2003), which computed the mean and Gaussian curvatures directly from a triangular mesh. In the following two sections, we briefly describe the methods used to compute the mean curvature and the Gaussian curvature.

The reason for using both the Gaussian curvature and the mean curvature for quantifying luminal surface irregularity is that the Gaussian curvature, as an intrinsic geometrical property, cannot be used to differentiate between regions curving inwards and those curving outwards. However, the mean curvature, as an extrinsic property, can be used for this purpose. Hemispheric regions curving inwards, such as the ulcers in the phantom experiment, have a positive mean curvature, whereas hemispheric regions curving in the opposite direction have a negative curvature (according to the orientation defined in equation (5)) (figure 2). Ulcers, as recesses on the vascular lumen, are located at regions where the luminal surface curves inwards, and therefore, must be associated with a positive mean curvature. Thus, in this study, we combined the Gaussian and mean curvatures in the identification of ulcers.

2.5.1. Discrete mean curvature. The mean curvature $\kappa_{H}$ at a point $P$ is related to the mean curvature normal operator $\mathbf{K}$ by the equation $\mathbf{K}(P)=2 \kappa_{H}(P) \mathbf{n}(P)$, where $\mathbf{n}(P)$ is the normal vector of the surface. Meyer et al (2003) proved that the integral of the mean curvature normal operator over a cell with vertex $i$ at the centre and with edge intersecting midpoints of the edges connecting the vertex $i$ and its 1-ring neighbour vertices (i.e., the grey region in figure 3(a)) can be expressed in terms of the angles of the triangulation and the length of the edges in the following simple form:

$$
\iint_{A} \mathbf{K}(x) \mathrm{d} A=\frac{1}{2} \sum_{j \in N_{1}(i)}\left(\cot \alpha_{i j}+\cot \beta_{i j}\right)\left(\mathbf{x}_{i}-\mathbf{x}_{j}\right),
$$




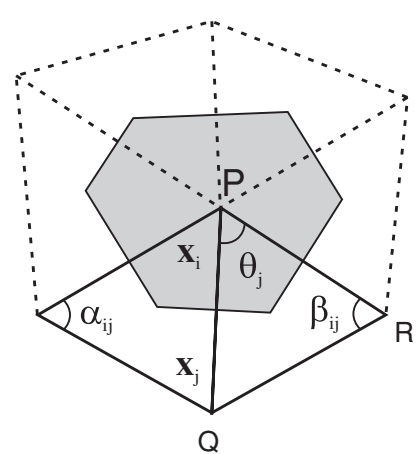

(a)

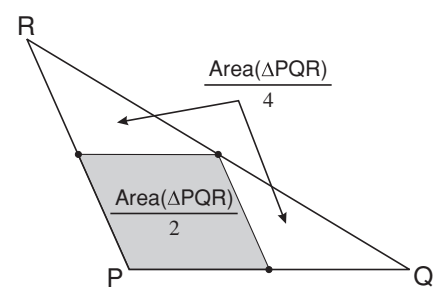

(c)

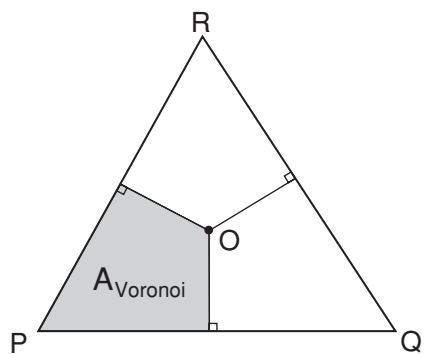

(b)

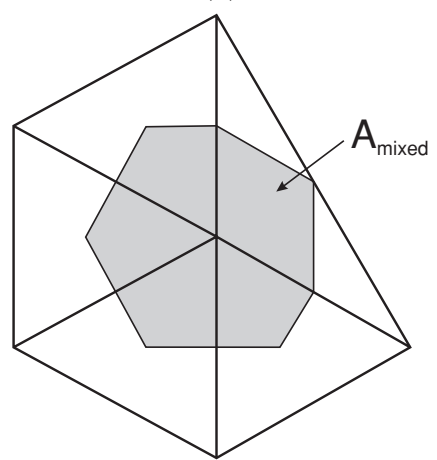

(d)

Figure 3. (a) Voronoi region (shaded) in a 1-ring neighborhood that does not contain a non-obtuse triangle. (b) Voronoi region (shaded) of $P$ on a non-obtuse triangle. (c) Voronoi region is not defined in an obtuse triangle. In this case, the shaded area is used for the surface partitioning purpose. (d) In a 1-ring neighbourhood with at least one obtuse triangle, the shaded area (with area $\left.A_{\text {mixed }}\right)$ is used for surface partitioning.

where $\alpha_{i j}$ and $\beta_{i j}$ are the two angles opposite to the edge in the two triangles sharing the edge $\left(\mathbf{x}_{i}, \mathbf{x}_{j}\right)$ and $N_{1}(i)$ is the set of 1-ring neighbour vertices of vertex $i$ (see figure 3(a)).

The only condition required for equation (1) to hold is that the edge of the cell centred at vertex $i$ intersects the midpoints of the set of edges $\left\{\left(\mathbf{x}_{i}, \mathbf{x}_{j}\right): j \in N_{1}(i)\right\}$. Thus, the grey region depicted in figure $3(\mathrm{a})$, called the Voronoi region, with its edge passing through the circumcentres of all triangles, is not the only region that satisfies this criterion. However, Meyer et al (2003) showed that the numerical error of curvature estimation is minimized when the Voronoi region is used. Given a non-obtuse triangle, the area of the Voronoi region for $P$ is (see figure 3(b)):

$$
A_{\text {Voronoi }}=\frac{1}{8}|P R|^{2} \cot (\angle Q)+\frac{1}{8}|P Q|^{2} \cot (\angle R) .
$$

The Voronoi partitioning scheme used for a non-obtuse triangle (shown in figure 3(a)) cannot be used for triangles with an obtuse angle (figure 3(c)), because the circumcentre of the obtuse triangle does not lie inside the triangle. However, as long as we use a region whose edges pass through the midpoint of the edges of the triangles, equation (1) is still valid. To allow efficient surface partitioning, Meyer et al (2003) used a third vertex that is the midpoint of the edge opposite to the obtuse angle to define the partition. This partition is represented 
by the grey region in figure 3(c). We can now define a new surface area for each vertex $\mathbf{x}$, denoted by $A_{\text {mixed }}(\mathbf{x})$, which is computed by the following algorithm (figure 3(d)):

$$
A_{\text {mixed }}(\mathbf{x})=\sum_{i=1}^{\# f} \operatorname{FA}\left(T_{i}\right)
$$

where

$$
\operatorname{FA}\left(T_{i}\right)= \begin{cases}A_{\text {Voronoi }}, & T_{i} \text { is non-obtuse. } \\ \operatorname{Area}\left(T_{i}\right) / 2, & T_{i} \text { is obtuse and the angle of } T_{i} \text { at } \mathbf{x} \text { is obtuse. } \\ \operatorname{Area}\left(T_{i}\right) / 4, & T_{i} \text { is obtuse and the angle of } T_{i} \text { at } \mathbf{x} \text { is non-obtuse }\end{cases}
$$

Here, \# $f$ denotes the number of triangular faces around the vertex $\mathbf{x}$ and $T_{i}$ denotes the triangle corresponding to the $i$ th face (see figure 3(a)).

With $A_{\text {mixed }}$ defined, we can express the mean curvature normal operator $\mathbf{K}$ as

$$
\mathbf{K}\left(\mathbf{x}_{i}\right)=\frac{1}{2 A_{\text {mixed }}\left(\mathbf{x}_{i}\right)} \sum_{j \in N_{1}(i)}\left(\cot \alpha_{i j}+\cot \beta_{i j}\right)\left(\mathbf{x}_{i}-\mathbf{x}_{j}\right) .
$$

Defining $\mathbf{n}$ to be the normal vector pointing inwards from the surface, the mean curvature is expressed as

$$
\kappa_{H}\left(\mathbf{x}_{i}\right)=\frac{1}{2} \mathbf{K}\left(\mathbf{x}_{i}\right) \cdot \mathbf{n}\left(\mathbf{x}_{i}\right)
$$

This equation implies that $\kappa_{H}>0$ if $\mathbf{K}$ points inwards from the surface. Otherwise, $\kappa_{H}<0$ (figure 2).

2.5.2. Discrete Gaussian curvature. The Gaussian curvature computation method is very similar to the way in which the mean curvature was obtained. First, we note from the Gauss-Bonnet theorem that the integral of the Gaussian curvature, $\kappa_{G}$, is

$$
\iint_{A} \kappa_{G} \mathrm{~d} A=2 \pi-\sum_{j=1}^{\# f} \theta_{j}
$$

where $\theta_{j}$ is the angle of the $j$ th face around the vertex $\mathbf{x}_{i}$ (see figure $3(\mathrm{a})$ ) and $\# f$ denotes the number of faces around the vertex.

We use the same partitioning scheme here as that used in the computation of the mean curvature normal operator giving the following expression for the Gaussian curvature:

$$
\kappa_{G}\left(\mathbf{x}_{i}\right)=\left(2 \pi-\sum_{j=1}^{\# f} \theta_{j}\right) / A_{\text {mixed }}\left(\mathbf{x}_{i}\right) .
$$

2.5.3. Smoothing of curvatures. Since semi-automatic or manual segmentation of US carotid images is prone to observer variability due to speckle, shadowing and noise (Mao et al 2000, Zagzebski 1996), we smooth the curvature (either the Gaussian or the mean curvature) at each vertex using an iterative algorithm proposed in Sundaram et al (2008) to successively average the curvature at a vertex with those of its adjacent neighbours. As emphasized in Sundaram et al (2008), the surface mesh was not smoothed in this procedure. Rather, only the curvature superimposed on the mesh was smoothed. We briefly summarize the smoothing algorithm 
as follows:

(i) initialize $S_{i, 0}=S_{i}$ and $\sigma_{0}=0.5 \mathrm{~mm}$,

(ii) at iteration $l+1$, set

$$
\begin{aligned}
& \sigma_{l+1}=1.1 \sigma_{l} \\
& S_{i, l+1}=\frac{1}{2}\left(S_{i, l}+\frac{\sum_{j \in N_{1}(i)} w_{i j, l} S_{j, l}}{\sum_{j \in N_{1}(i)} w_{i j, l}}\right)
\end{aligned}
$$

(iii) terminate when $\sigma_{l}>1 \mathrm{~mm}$,

where we have used the following notations:

$S_{i}=$ curvature at vertex $p_{i}$

$N_{i}=$ normal at vertex $p_{i}$

$N_{1}(i)=1$-ring neighbourhood of vertex $p_{i}$

$A_{i}=A_{\text {mixed }}\left(p_{i}\right)$.

We selected the termination condition to be $\sigma_{l}>1 \mathrm{~mm}$ because the segmentation inter-slice distance is $1 \mathrm{~mm} . w_{i j, l}$, the weight of the vertex $p_{j}$ in the calculation of the smoothed curvature at $p_{i}$ at iteration $l$, was obtained by the following equation:

$$
w_{i j, l}=A_{j}\left(N_{i} \cdot N_{j}\right) \frac{\exp \left(-\frac{\left\|p_{i}-p_{j}\right\|^{2}}{2 \sigma_{l}^{2}}\right)}{\sigma_{l}^{2}} .
$$

\subsection{Area-preserving flattened map}

For visualization and interpretation of the results, the surface curvature computed using the above method was superimposed on the arterial lumen to produce a 3D surface curvature map. Although 3D surface curvature maps thus constructed provide rich information on surface irregularity, the flattened representation of the 3D maps allows analysis in a single view, eliminating the need to study the 3D maps from multiple angles in an investigation, thereby allowing an easy comparison between two maps obtained either for a single patient at different time points or for different patients under different treatment arms. Thus, the area-preserving surface-flattening algorithm we developed in a previous publication (Chiu et al 2008a) was used for displaying the surface curvature maps for the phantom and the study subjects.

\subsection{Implementation}

The surface reconstruction (section 2.4), surface curvature computation (sections 2.5.1 and 2.5.2) and curvature smoothing algorithms (section 2.5.3) were implemented using $\mathrm{C}++$. The surface used in the phantom study contained approximately 3000 vertices. Reconstructing the phantom surface from $2 \mathrm{D}$ transverse contours took $0.8 \mathrm{~s}$. Computing $\kappa_{G}$ and $\kappa_{H}$ for the surface took $0.3 \mathrm{~s}$. Smoothing $\kappa_{G}$ and $\kappa_{H}$ took $9 \mathrm{~s}$. The implementation details of the areapreserving map were described elsewhere (Chiu et al 2008a) and are not repeated here. The carotid lumen surfaces used in the in vivo US studies contained approximately 500 vertices and required approximately $1 / 6$ of the time for the phantom study. All experiments were performed using an Intel ${ }^{\circledR}$ Core $^{\mathrm{TM}} 2.0 \mathrm{GHz}$ CPU with $1.0 \mathrm{~GB}$ memory. 
Table 1. Numerical accuracy in computing mean $\left(\kappa_{H}\right)$ and Gaussian curvature $\left(\kappa_{G}\right)$ for three spherical meshes, which were constructed by sampling a sphere with unity radius in different sampling intervals. For each mesh, the means and the root-mean-square errors (RMSE) of $\kappa_{H}$ and $\kappa_{G}$ were tabulated. Since a (continuous) sphere with unity radius has $\kappa_{H}$ and $\kappa_{G}$ both equalling 1, the means of $\kappa_{H}$ and $\kappa_{G}$ provide a measure of the bias in computing $\kappa_{H}$ and $\kappa_{G}$ for discrete spheres.

\begin{tabular}{ccllll}
\hline \multicolumn{5}{c}{ Sphere, radius $=1$} \\
\hline Ang. interval & No of points & Mean $\kappa_{H}$ & RMSE $\kappa_{H}$ & Mean $\kappa_{G}$ & RMSE $\kappa_{G}$ \\
\hline $5^{\circ}$ & 2450 & 1.0002 & $5.04 \times 10^{-4}$ & 1.0017 & $1.76 \times 10^{-3}$ \\
$10^{\circ}$ & 578 & 1.0007 & $1.89 \times 10^{-3}$ & 1.0070 & $7.01 \times 10^{-3}$ \\
$20^{\circ}$ & 128 & 1.0022 & $6.58 \times 10^{-3}$ & 1.0304 & $3.04 \times 10^{-2}$ \\
\hline
\end{tabular}

\subsection{Validation of numerical accuracy in the mean and Gaussian curvature calculations}

We evaluated the accuracy of the mean and Gaussian curvature computations by using two types of synthetic surfaces: the sphere and the torus. We also investigated the relationship between the number of sampling points used to represent the discrete surfaces and the numerical accuracy of the computed curvatures. These synthetic surfaces were not susceptible to noise, and thus, the curvature was not smoothed.

For a sphere, $\kappa_{G}$ equals $1 / r^{2}$ everywhere, where $r$ is the radius of the sphere, and $\kappa_{H}$ equals $1 / r$ (according to the orientation defined in equation (5)). We use the following parameterization for the sphere:

$$
(x, y, z)=(r \cos v \cos u, r \cos v \sin u, r \sin v),
$$

where $0 \leqslant u<2 \pi$ and $0 \leqslant v<\pi$. We created meshes of the unit sphere (i.e., $r=1$ ) by sampling $u$ and $v$ in $5^{\circ}, 10^{\circ}$ and $20^{\circ}$ intervals, and then computed $\kappa_{H}$ and $\kappa_{G}$ for each point on the meshes using the discrete curvature operators.

We also tested the curvature operators on a torus with the following parameterization:

$$
(x, y, z)=((a+r \cos u) \cos v,(a+r \cos u) \sin v, r \sin u),
$$

where $0 \leqslant u<2 \pi$ and $0 \leqslant v<2 \pi$. The theoretical $\kappa_{H}$ and $\kappa_{G}$ are expressed as follows:

$$
\begin{gathered}
\kappa_{H}=\frac{a+2 r \cos u}{2 r(a+r \cos u)}, \\
\kappa_{G}=\frac{\cos u}{r(a+r \cos u)} .
\end{gathered}
$$

We created three toroidal meshes (with $a=1, r=0.25$ ) by sampling $u$ and $v$ in $5^{\circ}, 10^{\circ}$ and $20^{\circ}$ intervals respectively, and then computed $\kappa_{H}$ and $\kappa_{G}$ for each point on the meshes.

\section{Results}

\subsection{Numerical accuracy of the mean and Gaussian curvature calculations}

Table 1 shows the root-mean-square error (RMSE) in the calculations of $\kappa_{H}$ and $\kappa_{G}$ for three spheres with different sampling intervals. Since a (continuous) sphere with unity radius has $\kappa_{H}$ and $\kappa_{G}$ both equalling 1 , the means of $\kappa_{H}$ and $\kappa_{G}$ provide a measure of the bias in computing $\kappa_{H}$ and $\kappa_{G}$ for discrete spheres, and therefore, were also tabulated. In this test, we observed, in 
Table 2. Numerical error in computing mean $\left(\kappa_{H}\right)$ and Gaussian curvature $\left(\kappa_{G}\right)$ for three toroidal meshes, which were constructed by sampling a torus (parameterized by equation (10) with $a=1$ and $r=0.25$ ) in different sampling intervals. For each mesh, the root-mean-square errors (RMSE) of $\kappa_{H}$ and $\kappa_{G}$ were tabulated.

\begin{tabular}{ccll}
\hline \multicolumn{4}{c}{ Torus, $a=1, r=0.25$} \\
\hline Ang. interval & No of points & RMSE $\kappa_{H}$ & RMSE $\kappa_{G}$ \\
\hline $5^{\circ}$ & 5184 & 0.00409 & 0.00727 \\
$10^{\circ}$ & 1440 & 0.00862 & 0.0181 \\
$20^{\circ}$ & 480 & 0.0233 & 0.0517 \\
\hline
\end{tabular}

agreement with Meyer et al (2003), that the integral of the discrete Gaussian curvature over an entire sphere is equal to $4 \pi$ regardless of the sampling interval used. For the spherical mesh that was produced by sampling $u$ and $v$ in $20^{\circ}$ intervals, because the spherical mesh has a surface area that is $3 \%$ smaller than the continuous sphere, the Gaussian curvature was overestimated by an average of $3 \%$ so that the Gaussian curvature of the discrete sphere integrates to $4 \pi$. Similar comment applies for the spheres with sampling intervals equalling $5^{\circ}$ and $10^{\circ}$. As the surface mesh becomes denser, the total surface area for the discrete sphere becomes closer to the continuous surface, resulting in a reduction of the RMSE of $\kappa_{G}$. Meanwhile, the RMSE of $\kappa_{H}$ also decreases as the sampling intervals of $u$ and $v$ decrease.

Table 2 shows the RMSE in the calculations of $\kappa_{H}$ and $\kappa_{G}$ for three tori with different sampling intervals. Similar to the results obtained for the spheres above, the integral of the discrete curvature over an entire torus agrees with the Gauss-Bonnet theorem (which is 0 for torus because torus has 1 hole), regardless of the sampling intervals of $u$ and $v$ in equation (10). The RMSEs of $\kappa_{H}$ and $\kappa_{G}$ converge to 0 as sampling intervals decrease.

\subsection{Mean and Gaussian curvatures of the vascular phantom}

Figure 4(a) shows the surface representing the vascular phantom, with the mean curvature colour-coded and superimposed on the surface. In this orientation, the $2 \mathrm{~mm}$ hemisphere was located on the top, with the $3 \mathrm{~mm}$ hemisphere located $11 \mathrm{~mm}$ below and the $4 \mathrm{~mm}$ hemisphere located further $14 \mathrm{~mm}$ below. The area-preserving flattened map of the vascular phantom surface shown in figure 4(a) was constructed and displayed in figure 4(b). The mean curvature values at all vertices were collected, and the 98th percentile of this sample was obtained. The clipping algorithm implemented in Visualization Toolkit (VTK) (Schroeder et al 2002) was used to extract regions with mean curvature greater than the 98th percentile. The result is displayed in figure 4(c), which shows the surface patches that have mean curvature greater than or equal to the 98th percentile, with the boundary of the flattened map represented by the black outline. Figure 4(c) shows that the hemispheres could not be detected as connected regions and that small patches outside the hemispheres were extracted erroneously. The mean curvature was then smoothed, colour-coded and superimposed on the vascular phantom surface, which is shown in figure 4(d). It is important to note that the same surface is shown in figures 4(a) and (d), only with different mean curvature values mapped onto them (i.e., the surface was not smoothed). The area-preserving flattened map of figure 4(d) is displayed in figure 4(e). We computed the 98th percentile of the 'smoothed' mean curvature and used it as the threshold to clip the surface, producing the result shown in figure 4(f). After smoothing, only regions corresponding to where the hemispheres were located were extracted, and the sizes of the three surface patches extracted corresponded approximately to the sizes of the hemispheres. 


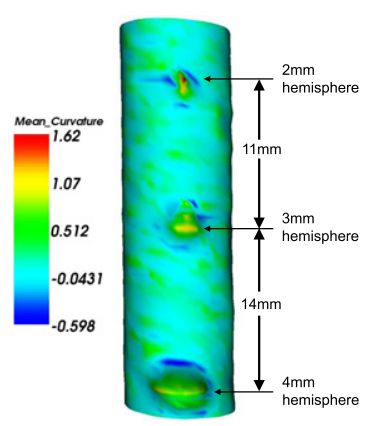

(a)

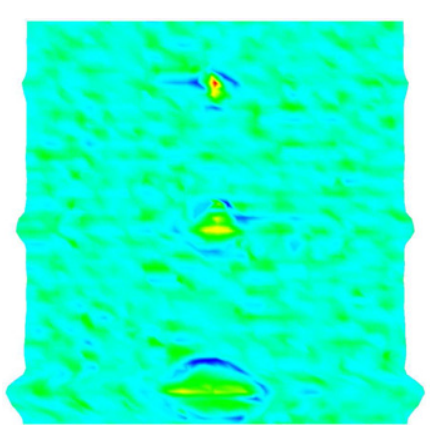

(b)

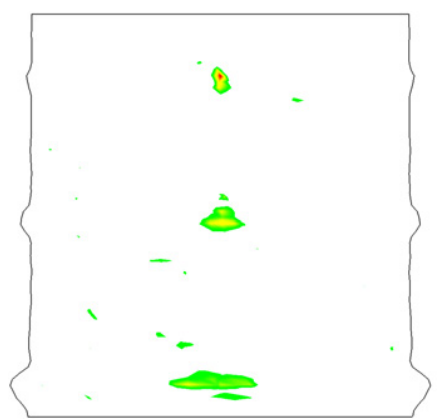

(c)

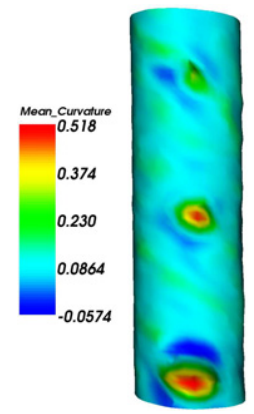

(d)

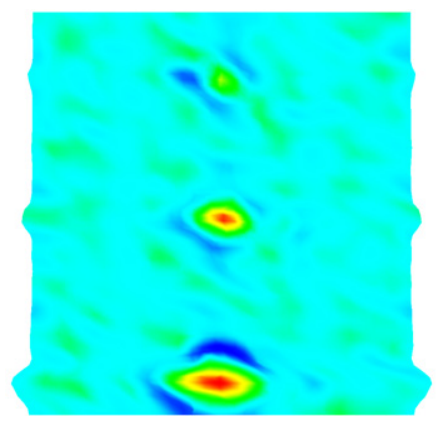

(e)

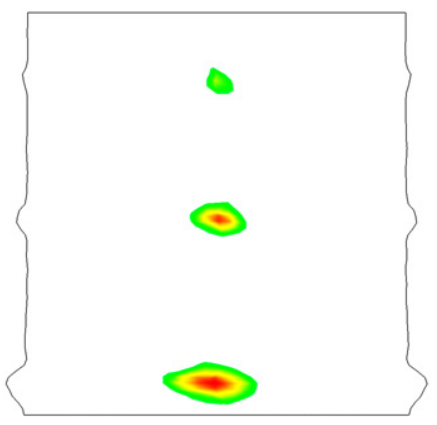

(f)

Figure 4. (a) Mean curvature map of the vascular phantom and (b) its flattened map. The 98th percentile of the mean curvature at all vertices was computed, which was used as a threshold to extract surface patches. (c) shows surface patches that have mean curvature greater than or equal to this threshold, with the boundary of the flattened surface represented by the black outline. (d) Smoothed mean curvature of the phantom and (e) its flattened map. (f) Surface patches that have mean curvature greater than or equal to the 98th percentile.

The Gaussian curvature was computed, colour-coded and superimposed on the vascular phantom as shown in figure 5(a). The experiment performed here was the same as that described previously for the mean curvature. The area-preserving flattened map of figure 5(a) was displayed in figure 5(b). Figure 5(c) shows regions with the Gaussian curvature that was greater than or equal to the 98th percentile. The Gaussian curvature was then smoothed (figure 5(d)) and flattened (figure 5(e)). Figure 5(f) shows regions with the 'smoothed' Gaussian curvature greater than or equal to the 98th percentile. The results obtained were similar to those for the mean curvature.

\subsection{Results for study subjects}

We demonstrated in the phantom experiment that ulcers are characterized by high Gaussian and mean curvatures. In the phantom experiment, we identified ulcers using the Gaussian and mean curvatures independently because there is no outward-curving region in the vascular phantom. However, for accurate identification of ulcers from carotid luminal surfaces of human subjects, we required to combine the use of the Gaussian and mean curvatures because it is not possible to differentiate between inward- and outward-curving regions by using the Gaussian curvature alone (figure 2). Outward-curving regions exist in carotid luminal surfaces, 


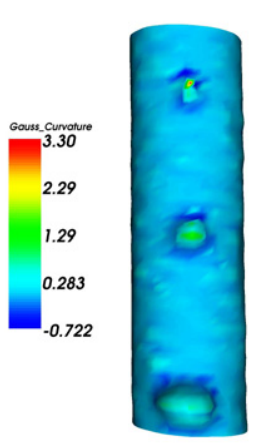

(a)

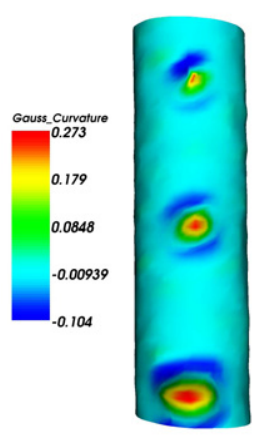

(d)

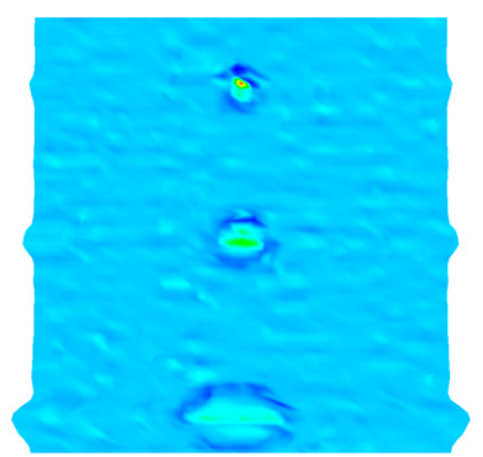

(b)

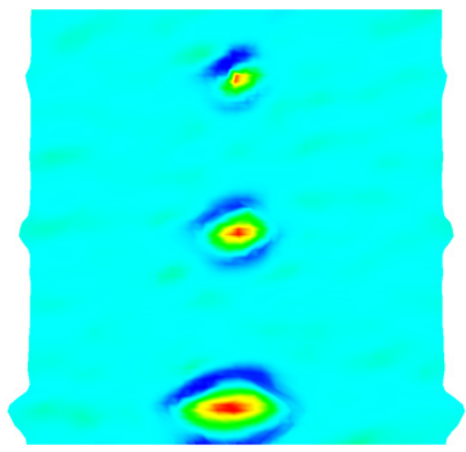

(e)

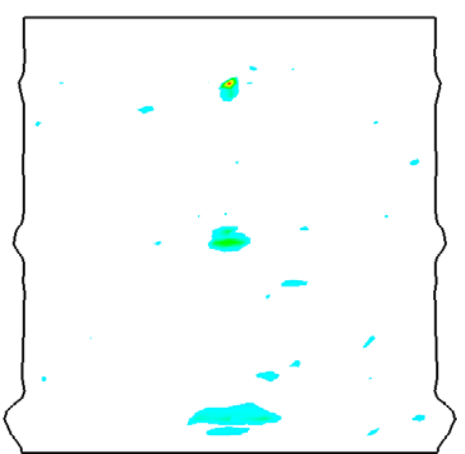

(c)

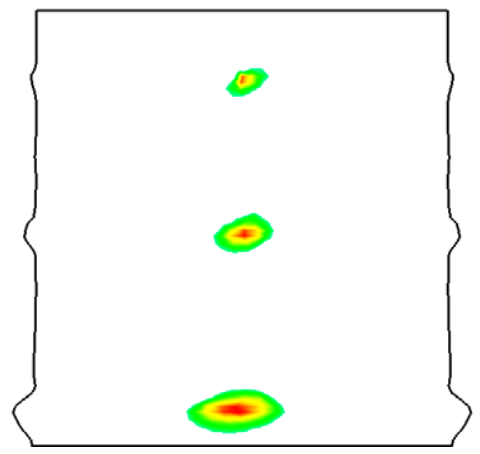

(f)

Figure 5. (a) Gaussian curvature map of the vascular phantom and (b) its flattened map. The 98th percentile of the Gaussian curvature at all vertices was computed, which was used as a threshold to extract surface patches. (c) shows surface patches that have Gaussian curvature greater than or equal to this threshold, with the boundary of the flattened surface represented by the black outline. (d) Smoothed Gaussian curvature of the phantom and (e) its flattened map. (f) Surface patches that have Gaussian curvature greater than or equal to the 98th percentile.

and the combined information of the Gaussian and mean curvatures allowed us to exclude these regions in the identification of ulcers.

Figure 6(a) shows the 3D carotid US image of subject 1 with segmented lumen. Figure 6(b) shows the CCA lumen surface of subject 1 with the smoothed Gaussian curvature colourcoded and superimposed on the surface. Figures 6(c)-(e) show the transverse 2D images that correspond to slices 1, 2 and 3 labelled in figure 6(b). Figure 6(f) shows the area-preserving flattened map with the Gaussian curvature colour-coded and superimposed on the map. The shaded area in this map represents regions where the Gaussian curvature was greater than or equal to the 98th percentile (as described in the phantom experiment). We denoted this region by $G_{98}$. Figure $6(\mathrm{~g})$ shows the area-preserving flattened map with the mean curvature colour-coded and superimposed on the map. Similarly, the shaded area in this map represents regions where the mean curvature was greater than or equal to the 98th percentile, denoted by $M_{98}$. As a criterion, we identify ulcers as the intersection between $G_{98}$ and $M_{98}$. Figure 6(h) shows the region identified by this method.

Figure 7(a) shows the 3D carotid US image of subject 2 with segmented lumen. Figure 7(b) shows the CCA lumen surface of subject 2 with the smoothed Gaussian curvature 


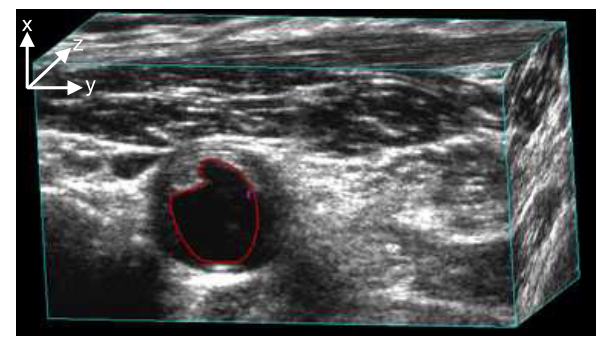

(a)

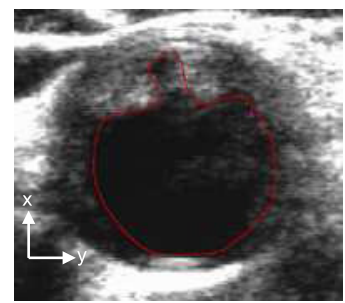

(c)

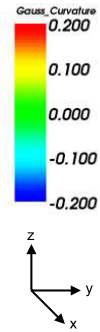

$\searrow$

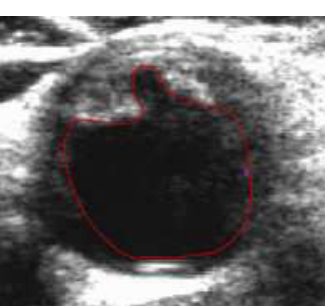

(d)

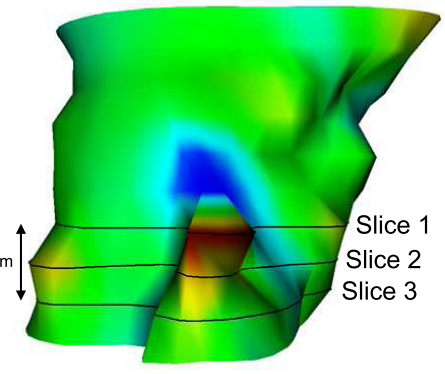

(b)

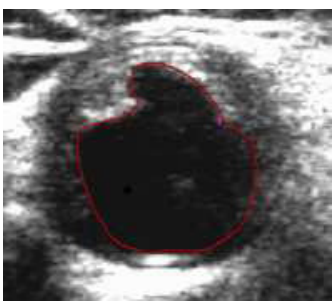

(e)

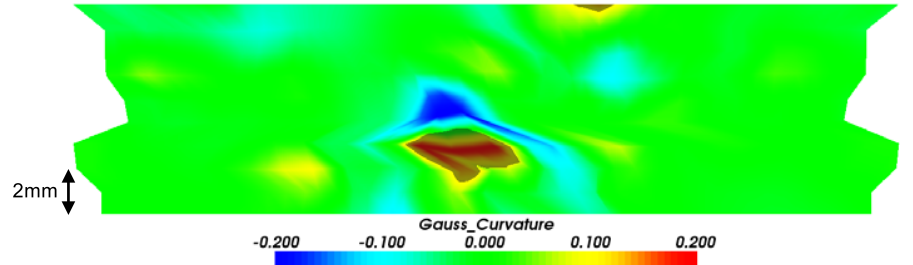

(f)

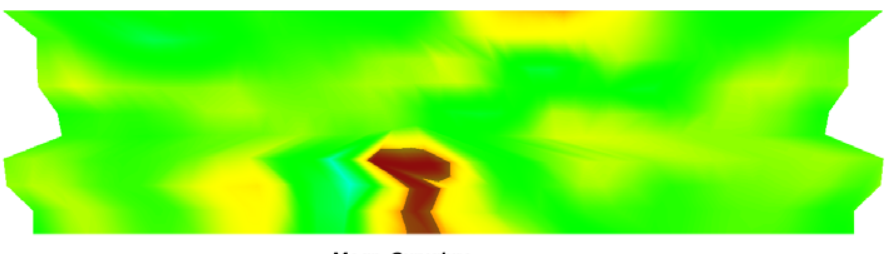

$\begin{array}{lll}-0.500 & -0.250 & \text { Mean_Curvature } \\ 0.000 & 0.250\end{array}$

$(\mathrm{g})$

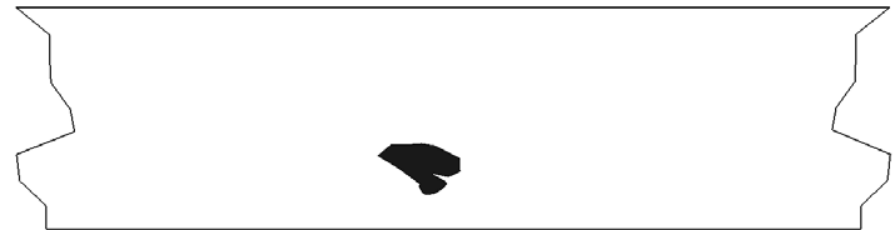

(h)

Figure 6. (a) 3D US image with segmented lumen. (b) 3D Gaussian curvature map for the arterial lumen of subject 1. The transverse views of (c) slice 1, (d) slice 2 and (e) slice 3 as labelled in (b). (f) 2D Gaussian curvature map corresponding to the 3D map shown in (b). The shaded area represents the region where the Gaussian curvature is equal to or greater than the 98th percentile (i.e., $G_{98}$.) (g) 2D mean curvature map corresponding to the 3D map shown in (b). The shaded area represents the region where the mean curvature is equal to or greater than the 98 th percentile (i.e., $\left.M_{98}\right)$. (h) $G_{98} \bigcap M_{98}$. 


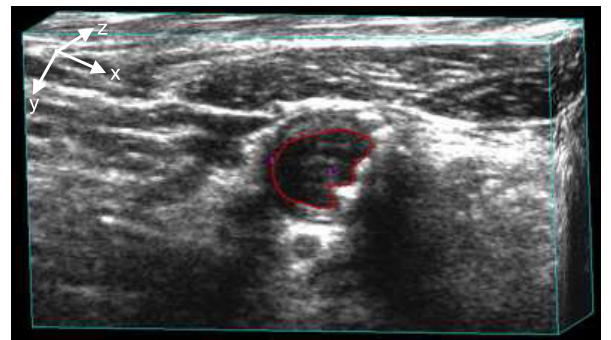

(a)

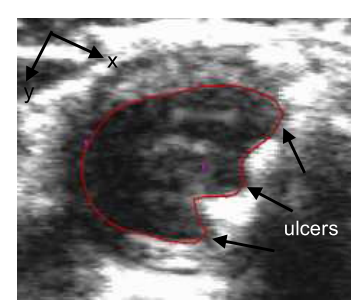

(c)

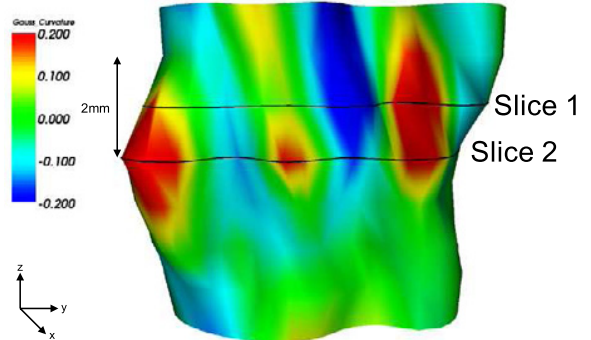

(b)

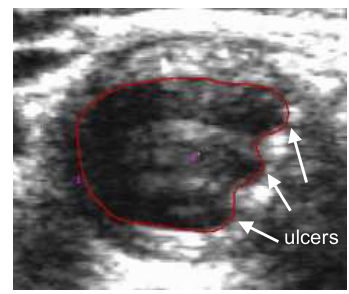

(d)

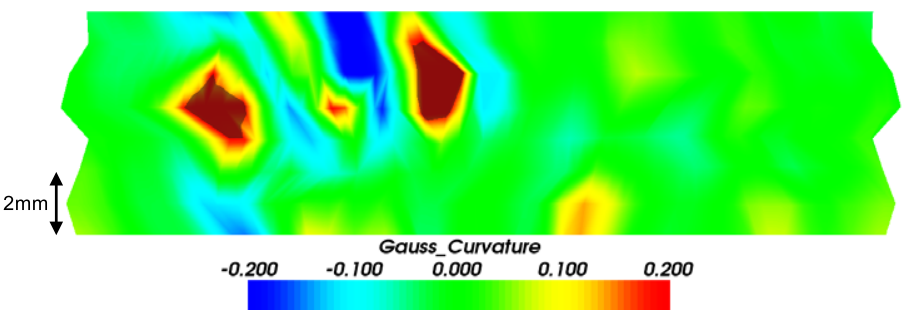

(e)

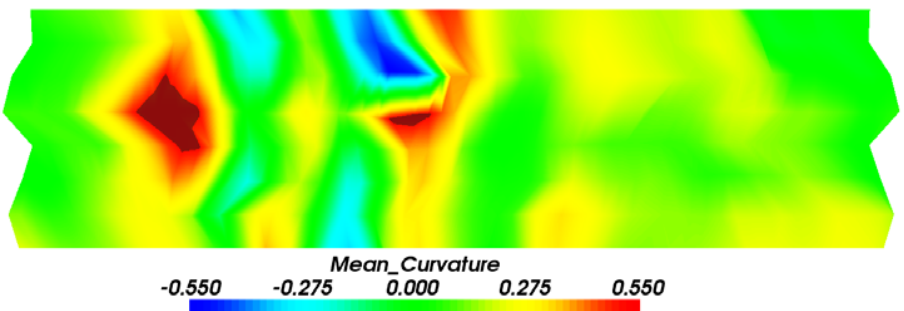

(f)

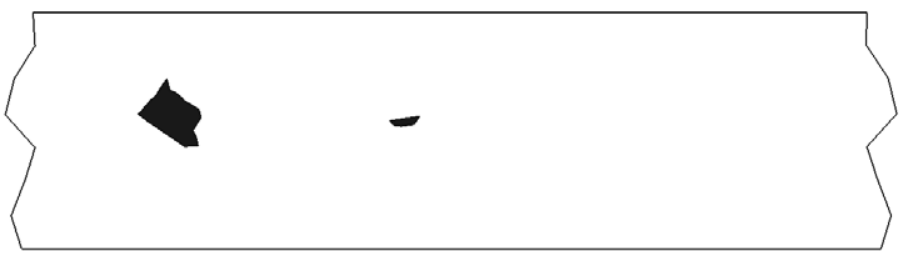

(g)

Figure 7. (a) 3D US image with segmented lumen. (b) 3D Gaussian curvature map for the arterial lumen of subject 2. The transverse views of (c) slice 1 and (d) slice 2 as labelled in (b). (e) 2D Gaussian curvature map corresponding to the 3D map shown in (b). The shaded area represents the region where the Gaussian curvature is equal to or greater than the 98th percentile (i.e., $G_{98}$.) (f) 2D mean curvature map corresponding to the 3D map shown in (b). The shaded area represents the region where mean curvature is equal to or greater than the 98th percentile (i.e., $M_{98}$ ). (g) $G_{98} \bigcap M_{98}$. 
colour-coded and superimposed on the surface. Figures 7(c) and (d) show the transverse 2D images that correspond to slices 1 and 2 labelled in figure 7(b). Unlike subject 1, there were multiple smaller ulcers on the artery of this subject. Figure 7(e) shows the area-preserving flattened map with the Gaussian curvature colour-coded and superimposed on the map. The shaded area represents the region $G_{98}$. We note from this figure that $G_{98}$ covered regions where two of the three ulcers were, but did not cover the ulcer between the two identified ulcers. In addition, the ulcer on the right of figure 7(e) is much smaller than the detected region. The upper part of the detected region curves outwards, and therefore, is not a part of the ulcer. The lower part of the detected region, however, corresponds to an ulcer shown in figure 7(d). Figure 7(f) shows the area-preserving flattened map with the mean curvature colour-coded and superimposed on the map. The shaded area represents the region $M_{98}$. The region we mentioned above that curves outwards had a negative mean curvature, and thus, was not covered by $M_{98}$. Figure $7(\mathrm{~g})$ shows the region $G_{98} \bigcap M_{98}$, which covered two of the three ulcers.

\section{Discussion and conclusion}

In this work, we have introduced a multi-step method for quantifying and displaying the irregularity of vessel wall and plaque surfaces. First, we implemented the method proposed by Meyer et al (2003) to compute the Gaussian and mean curvatures. We validated our curvature computations by testing the algorithm using discrete spherical and toroidal meshes sampled in different intervals, and have shown that the RMSEs for the Gaussian and mean curvature computations were small if the meshes were densely sampled. Since ultrasound images are susceptible to artefacts caused by speckle, shadowing and noise (Mao et al 2000, Zagzebski 1996), we applied a smoothing algorithm proposed in Sundaram et al (2008) to smooth the curvatures computed for surfaces segmented from 3D US images. It was demonstrated in the vascular phantom experiment that the $2 \mathrm{~mm}, 3 \mathrm{~mm}$ and $4 \mathrm{~mm}$ ulcers could be detected as connected regions after the curvatures had been smoothed (figures 4(f) and 5(f)).

In our phantom study, the ulcers were oriented in a direction that was facing the transducer. It is known that sections of boundary perpendicular to the direction of the pulse transmission produce higher contrast, and sections of boundary parallel to the pulse transmission direction give lower contrast (Rohling et al 1998). Therefore, the segmentation of the ulcers may be less accurate if the boundaries of the ulcers are parallel, instead of perpendicular, to the direction of US propagation. The same may be true for the in vivo US images, causing the ulcers with boundaries parallel to the direction of US transmission more difficult to be detected. Compound US imaging is a potential solution to this problem (Rohling et al 1998, Jespersen et al 2000), in which multiple US images of the vessel are obtained from different directions, then averaged and registered to produce the final US image.

To enhance the visualization and facilitate the interpretation of the 3D surface curvature maps (i.e., the luminal surface with curvatures colour-coded and mapped onto it), the 3D maps were flattened to 2D using the area-preserving flattening map algorithm introduced in a previous publication (Chiu et al 2008a). 2D maps allow the study of surface curvature maps in a single view, thereby allowing an easy comparison between multiple maps. For example, the difference between figures 4(c) and (f) (or figures 5(c) and (f)) was much easier to visualize in 2D. Finally, we combined the Gaussian and mean curvatures in detecting ulcers from luminal surfaces segmented from 3D US images acquired for two human subjects. Only regions with high Gaussian and mean curvatures were identified as ulcers. These two criteria precluded the false detection of outward-curving regions as ulcers, since outward-curving regions would have a negative mean curvature. 
This study has shown the accuracy and practicality of the proposed algorithm in the synthetic surface experiment and the vascular phantom experiment, respectively. In demonstrating the application of the proposed algorithm for the carotid luminal surfaces of human subjects, we considered a physician's identification of the ulcers as the surrogate gold standard, and have segmented the arterial lumen according to his identification. One limitation of this study is that the ulcer identification and the subsequent segmentation are subjective, and therefore, susceptible to observer bias and variability. Future studies should be performed to quantify the intra- and inter-observer variability in luminal segmentation. The accuracy of the segmented luminal boundaries should also be validated against the corresponding histological specimens before the proposed ulcer detection algorithm is applied. After an ulcer has been detected using the algorithm, the location of the detected ulcers should also be verified against histological observations in future studies.

The motivation for this work stems from increasing evidences that suggest the risk of vascular events is related to atherosclerosis plaque stability (Barnett 1992, Warlow 1991). The risk of stroke has been correlated with carotid stenosis (NASCET Collaborators 1991, Warlow 1991) and with plaque features related to plaque stability, such as lesion surface irregularities, ulcerations and fissures (Eliasziw et al 1994, Streifler et al 1994, Rothwell et al 2000, Fisher et al 2005). An automated method described here may be used to provide a rapid and feasible method of screening a large number of images for the presence of surface irregularities. Carotid US images with irregular surface features can then be flagged for observer analysis and validation. The proposed automatic algorithm can be used in large cross-sectional studies to evaluate the risk associated with irregular plaque surfaces and in longitudinal studies to evaluate whether there is any correlation between the degree of plaque surface irregularity and symptoms of ICA occlusive diseases (e.g., transient ischemic attacks and strokes), which are two important long-term goals for vascular imaging research.

\section{Acknowledgments}

The authors would like to thank A Mendel for constructing the vascular phantom used in this experiment. The authors gratefully acknowledge the financial support provided by the Canadian Institute of Health Research and the Ontario R\&D Challenge Fund. A Fenster holds a Canada Research Chair in Biomedical Engineering, and acknowledges the support of the Canada Research Chair Program. B Chiu acknowledges the support of the Ontario Graduate Scholarship.

\section{References}

Ainsworth C D, Blake C C, Tamayo A, Beletsky V, Fenster A and Spence J D 2005 3D ultrasound measurement of change in carotid plaque volume: a tool for rapid evaluation of new therapies Stroke 36 1904-9

Barnett H J 1992 Stroke prevention by surgery for symptomatic disease in carotid territory Neurol. Clin. 10 281-92

Besl P J and Jain R C 1986 Invariant surface characteristics for 3D object recognition in range images Comput. Vis. Graph. Image Process. 33 33-80

Chiu B, Egger M, Spence J D, Parraga G and Fenster A 2006 Quantification of carotid vessel atherosclerosis Proc. SPIE $614361430 \mathrm{~B}$

Chiu B, Egger M, Spence J D, Parraga G and Fenster A 2008a Area-preserving flattening maps of 3D ultrasound carotid arteries images Med. Image Anal. 12 676-88

Chiu B, Egger M, Spence J D, Parraga G and Fenster A 2008b Quantification of carotid vessel wall and plaque thickness change using 3D ultrasound images Med. Phys. 35 3691-710

de Bray J M, Baud J M and Dauzat M 1997 Consensus concerning the morphology and the risk of carotid plaques Cerebrovasc. Dis. 7 289-96

do Carmo M P 1976 Differential Geometry of Curves and Surfaces (Englewood Cliffs, NJ: Prentice-Hall) 
Eliasziw M, Streifler J Y, Fox A J, Hachinski V C, Ferguson G G and Barnett H J 1994 Significance of plaque ulceration in symptomatic patients with high-grade carotid stenosis. North American Symptomatic Carotid Endarterectomy Trial Stroke 25 304-8

Fenster A, Downey D B and Cardinal H N 2001 Three-dimensional ultrasound imaging Phys. Med. Biol. 46 R67-99

Fenster A, Landry A, Downey D B, Hegele R A and Spence J D 2004 3D ultrasound imaging of the carotid arteries Curr. Drug Target Cardiovasc. Haematol. Disord. 4 161-75

Fisher M, Paganini-Hill A, Martin A, Cosgrove M, Toole J F, Barnett H J and Norris J 2005 Carotid plaque pathology: thrombosis, ulceration, and stroke pathogenesis Stroke 36 253-7

Golledge J, Greenhalgh R M and Davies A H 2000 The symptomatic carotid plaque Stroke 31 774-81

Han C, Hatsukami T S and Yuan C 2001 Accurate lumen surface roughness measurement method in carotid atherosclerosis Proc. SPIE 4322 1817-27

Jespersen S K, Wilhjelm J E and Sillesen H 2000 In vitro spatial compound scanning for improved visualization of atherosclerosis Ultrasound Med. Biol. 26 1357-62

Ladak H M, Mao F, Wang Y, Downey D B, Steinman D A and Fenster A 2000 Prostate boundary segmentation from 2D ultrasound images Med. Phys. 27 1777-88

Landry A and Fenster A 2002 Theoretical and experimental quantification of carotid plaque volume measurements made by three-dimensional ultrasound using test phantoms Med. Phys. 29 2319-27

Landry A, Spence J D and Fenster A 2005 Quantification of carotid plaque volume measurements using 3D ultrasound imaging Ultrasound Med. Biol. 31 751-62

Lobregt S and Viergever M A 1995 A discrete dynamic contour model IEEE Trans. Med. Imaging 14 12-24

Mao F, Gill J, Downey D and Fenster A 2000 Segmentation of carotid artery in ultrasound images: method development and evaluation technique Med. Phys. 27 1961-70

Meairs S and Hennerici M 1999 Four-dimensional ultrasonographic characterization of plaque surface motion in patients with symptomatic and asymptomatic carotid artery stenosis Stroke 30 1807-13

Meyer M, Desbrun M, Schroder P and Barr A H 2003 Visualization and Mathematics III 1st edn (Heidelberg: Springer) pp 35-57

Miskolczi L, Guterman L R, Flaherty J D and Hopkins L N 1996 Depiction of carotid plaque ulceration and other plaque-related disorders by intravascular sonography: a flow chamber study AJNR Am. J. Neuroradiol. 17 1881-90

NASCET Collaborators 1991 Beneficial effect of carotid endarterectomy in symptomatic patients with high-grade stenosis N. Engl. J. Med. 325 445-53

Rickey D W, Picot P A, Christopher D A and Fenster A 1995 A wall-less vessel phantom for doppler ultrasound studies Ultrasound Med. Biol. 21 1163-76

Rohling R N, Gee A H and Berman L 1998 Automatic registration of 3D ultrasound images Ultrasound Med. Biol. 24 841-54

Rothwell P M, Gibson R and Warlow C P 2000 Interrelation between plaque surface morphology and degree of stenosis on carotid angiograms and the risk of ischemic stroke in patients with symptomatic carotid stenosis Stroke 31 615-21

Schminke U, Motsch L, Hilker L and Kessler C 2000 Three-dimensional ultrasound observation of carotid artery plaque ulceration Stroke 31 1651-5

Schroeder W J, Martin K and Lorensen W 2002 The Visualization Toolkit, an Object-Oriented Approach to 3D Graphics (New York, NY: Kitware)

Sitzer M, Muller W, Rademacher J, Siebler M, Hort W, Kniemeyer H W and Steinmetz H 1996 Colour-flow doppler-assisted duplex imaging fails to detect ulceration in high-grade internal carotid artery stenosis J. Vasc. Surg. 23 461-5

Sitzer M, Muller W, Siebler M, Hort W, Kniemeyer H W, Jancke L and Steinmetz H 1995 Plaque ulceration and lumen thrombus are the main sources of cerebral microemboli in high-grade internal carotid artery stenosis Stroke 26 1231-3

Spence J D, Tamayo A, Lownie S P, Ng W P and Ferguson G G 2005 Absence of microemboli on transcranial doppler identifies low-risk patients with asymptomatic carotid stenosis Stroke 36 2373-8

Steinke W, Hennerici M, Rautenberg W and Mohr J P 1992 Symptomatic and asymptomatic high-grade carotid stenoses in doppler colour-flow imaging Neurology 42 131-8

Streifler J Y, Eliasziw M, Fox A J, Benavente O R, Hachinski V C, Ferguson G G and Barnett H J 1994 Angiographic detection of carotid plaque ulceration. Comparison with surgical observations in a multicentre study. North American Symptomatic Carotid Endarterectomy Trial Stroke 25 1130-2

Sundaram P, Zomorodian A, Beaulieu C and Napel S 2008 Colon polyp detection using smoothed shape operators: preliminary results Med. Image Anal. 12 99-119 
Sztajzel R 2005 Ultrasonographic assessment of the morphological characteristics of the carotid plaque Swiss Med. Wkly. 135 635-43

The Heart and Stroke Foundation of Canada 2000 The Changing Face of Heart Disease and Stroke in Canada http://dsp-psd.pwgsc.gc.ca/Collection/H88-3-30-2001/pdfs/age/face_e.pdf

The Heart and Stroke Foundation of Canada 2003 The Growing Burden of Heart Disease and Stroke in Canada http://www.cvdinfobase.ca/cvdbook/CVD_En03.pdf

Thom T et al 2006 Heart disease and stroke statistics-2006 update: a report from the American Heart Association Statistics Committee and Stroke Statistics Subcommittee Circulation 113 e85-151

Warlow C 1991 MRC European Carotid Surgery Trial: interim results for symptomatic patients with severe (70-99\%) or with mild (0-29\%) carotid stenosis Lancet 337 1235-43

Zagzebski J A 1996 Essentials of Ultrasound Physics (St. Louis, MO: Mosby) 